\title{
Guanosine Quadruplexes in Solution: A Small-Angle X-Ray Scattering Analysis of Temperature Effects on Self-Assembling of Deoxyguanosine Monophosphate
}

\author{
P. Mariani, ${ }^{1}$ F. Spinozzi, ${ }^{1}$ F. Federiconi, ${ }^{1}$ M. G. Ortore, ${ }^{1}$ H. Amenitsch, ${ }^{2}$ \\ L. Spindler, ${ }^{3}$ and I. Drevensek-Olenik ${ }^{4}$ \\ ${ }^{1}$ Physical Science Section, Research Unit of Ancona, SAIFET Department, CNISM, Marche Polytechnic University, \\ Via Brecce Bianche, 60131 Ancona, Italy \\ ${ }^{2}$ Institute of Biophysics and Nanosystems Research, Austrian Academy of Sciences, Schmiedlstrasse 6, 8042 Graz, Austria \\ ${ }^{3}$ Faculty of Mechanical Engineering, University of Maribor, Smetanova 17, SI 2000 Maribor, and J. Stefan Institute, Jamova 39, \\ SI 1000 Ljubljana, Slovenia \\ ${ }^{4}$ Faculty of Mathematics and Physics, University of Ljubljana, Jadranska 19, and J. Stefan Institute, Jamova 39, \\ SI 1000 Ljubljana, Slovenia
}

Correspondence should be addressed to P. Mariani, mariani@univpm.it

Received 26 January 2010; Accepted 26 March 2010

Academic Editor: Mateus Webba da Silva

Copyright () 2010 P. Mariani et al. This is an open access article distributed under the Creative Commons Attribution License, which permits unrestricted use, distribution, and reproduction in any medium, provided the original work is properly cited.

\begin{abstract}
We investigated quadruplex formation in aqueous solutions of $2^{\prime}$-deoxyriboguanosine $5^{\prime}$-monophosphate, $d(p G)$, which takes place in the absence of the covalent axial backbone. A series of in-solution small angle X-ray scattering experiments on $\mathrm{d}(\mathrm{pG}) \mathrm{have}$ been performed as a function of temperature in the absence of excess salt, at a concentration just above the critical one at which self-assembling occurs. A global fit approach has been used to derive composition and size distribution of the scattering particles as a function of temperature. The obtained results give thermodynamical justification for the observed phase-behavior, indicating that octamer formation is essential for quadruplex elongation. Our investigation shows that $\mathrm{d}(\mathrm{pG})$ quadruplexes are very suitable to assess the potential of G-quadruplex formation and to study the self-assembling thermodynamics.
\end{abstract}

\section{Introduction}

It is well known that guanosine-rich single stranded sequences of DNA, as found in telomeres and in other parts of the genome, especially in promoters $[1,2]$, can adopt various tertiary structures, including G-quadruplexes. Gquadruplexes are four-stranded helical structures, made by the stacking of planar quartets (also indicated as tetramers), arising from Hoogsten hydrogen-bonding between four guanines $(G)$. The biological role of such sequences and the structural properties of G-quadruplexes have been extensively discussed $[1,3-6]$, and several reviews, focusing mainly on their topology [7-11] or on telomerase activity [12], have been published. However, the understanding of basic physical properties is still rather limited, even for short sequences comprising only 3 or 4 quartets. In particular, the mechanisms and the principles that govern quadruplex formation and stability in terms of sequence space and counter-ion effects, as expressed by thermodynamic and kinetic parameters, are still unknown.

Hence, in order to assess the potential of G-quadruplex formation and possible biological roles, the in vitro thermodynamic and kinetic properties of guanosine-rich sequences need to be investigated. The $2^{\prime}$-deoxyriboguanosine $5^{\prime}$ monophospate, $\mathrm{d}(\mathrm{pG})$, can be regarded as a useful model system for self-assembling studies. In fact, despite the absence of the sugar-phosphate axial backbone, $d(p G)$ in aqueous solutions and in the presence of the proper counterion forms quadruplexes [13]. X-ray and neutron diffraction experiments showed that $\mathrm{d}(\mathrm{pG})$ quartets are stacked on the top of each other at the van der Waals distance of $3.4 \AA$ and rotated with respect to each other by an angle of about $30^{\circ}$ 
[14-16]. The presence of monovalent cations was observed to be essential for the stability of these supramolecular aggregates: the cation, located between two G-quartets, stabilizes the hydrogen-bonded quartets by cation-dipole interactions with the O6 ketone groups of eight separate molecules of guanine, enhancing base-stacking interactions [17].

Depending on the concentration, $\mathrm{d}(\mathrm{pG})$ quadruplexes in water form cholesteric and hexagonal phases [13, 18, 19]. Extended analysis showed that the phase behavior depends on the length of the quadruplexes and on the nature and concentration of counterions [17-23]. Moreover, phase transitions and preferential (quadruplex lateral or axial) hydration were detected when high-pressure effects were considered [16]. Accordingly, temperature was suggested to induce quadruplex fragmentation [13].

The pathway that in dilute conditions governs the formation of $\mathrm{d}(\mathrm{pG})$ quadruplexes has been described as follows (see also Figure 1) [22, 24]:

$$
\begin{gathered}
4 \mathrm{G}+\mathrm{M} \rightleftharpoons \mathrm{G}_{4} \mathrm{M} \\
2 \mathrm{G}_{4} \mathrm{M} \rightleftharpoons \mathrm{G}_{8} \mathrm{M}+\mathrm{M} \\
\mathrm{G}_{8} \mathrm{M}+\mathrm{G}_{4} \mathrm{M} \rightleftharpoons \mathrm{G}_{12} \mathrm{M}_{2} \\
\cdots \\
\mathrm{G}_{(n-1) 4} \mathrm{M}_{(n-2)}+\mathrm{G}_{4} \mathrm{M} \rightleftharpoons \mathrm{G}_{4 n} \mathrm{M}_{(n-1)}
\end{gathered}
$$

where $\mathrm{M}$ indicates the monovalent counter-ion and the index on the $G$ symbol represents the aggregation state of $d(p G)$ molecules ( $\mathrm{G}$ is for monomer, $\mathrm{G}_{4}$ for $\mathrm{G}$-quartet, $\mathrm{G}_{8}$ for dimer of G-quartets, etc.; note that in $\mathrm{G}_{4 n}, n$ corresponds to the number of stacked quartets).

The first step is related to the quartet formation, which has been suggested to be strongly favored [20]. In the second step, a dimer of tetramers forms, associated with the releasing of one counter-ion. The formation of quadruplexes results from the successive addition of $\mathrm{G}$-quartets to $i$-mer fragments, as indicated in (1c) and (1d). In these processes, no counter-ions are released.

The self-assembling of $\mathrm{d}(\mathrm{pG})$, in the form of ammonium salt, has been recently studied by in-solution small angle $\mathrm{X}$-ray scattering (SAXS), both in the presence and in the absence of excess potassium ions [24]. A global fit analysis showed that the process is strongly modified by the excess potassium in solution, in full agreement with previous dynamic light scattering (DLS) and ${ }^{31} \mathrm{P} N M R$ measurements [25-27]. Accordingly, only a few aggregate forms (namely, G-quartets, G-octamers and monodisperse long G-quadruplexes) were detected in solution. In particular, SAXS experiments performed as a function of $\mathrm{d}(\mathrm{pG})$ concentration showed that the G-quartet-based selfassembling occurs above a critical guanosine concentration, $c_{0}$, of $4.5 \mathrm{wt} \%$. Indeed, at this concentration, quadruplexes and G-octamers became detectable. Interestingly, the length of quadruplexes was observed to continuously increase with increasing concentration ( $n$ increases from 6 to 40 when concentration increases from 4.5 to $10 \mathrm{wt} \%$ [24]): as the amount of $\mathrm{G}_{8}$ was detected to remain quite constant,
(A)

A)
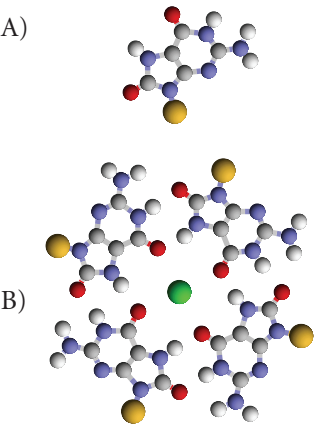

(B')

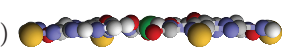

(C)

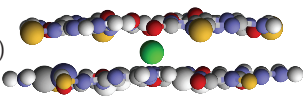

(D)

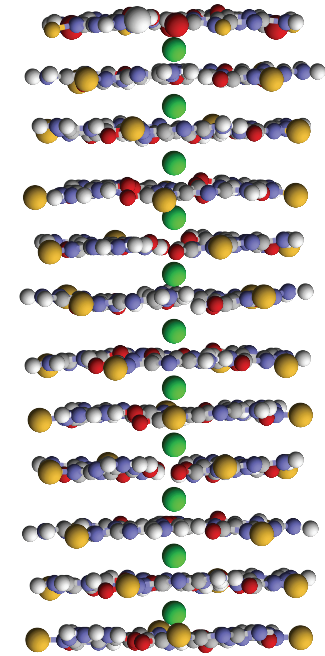

Figure 1: Representation of the aggregates formed by $\mathrm{d}(\mathrm{pG})$ in water (yellow and green beads represent the sugar-phosphate residue and the monovalent cation, resp.). (A) guanosine molecule; (B) and ( $\left.\mathrm{B}^{\prime}\right)$ arrangement of four guanosine residues in a G-quartet (top and lateral views); $\mathrm{K}^{+}, \mathrm{Na}^{+}$, and $\mathrm{NH}_{3}{ }^{+}$have the right size to occupy the tetramer inner cavity; (C) lateral view of the G-octamer, formed by two stacked quartets; the cation is located between the two quartets; (D) lateral view of the G-quadruplex, formed by stacking of discrete cation-bound quartets.

elongation was associated with a decrease of concentration of both free guanosine monomers and G-quartets. Excess $\mathrm{K}^{+}$in solution was observed to strongly induce quadruplex growth: indeed, very long aggregates form even below the critical concentration $c_{0}$ (at $0.2 \mathrm{M} \mathrm{KCl}, n$ around 160 was observed for $c=4 \mathrm{wt} \%$ [24]).

The thermodynamics of the process was explained in the framework of a nucleation-elongation model, where an unfavorable nucleation step, leading to G-octamers, is followed by a favorable spontaneous elongation, which rapidly progresses once a stable nucleus (the dodecamer, $\mathrm{G}_{12}$ ) is achieved. Very interesting, no $\mathrm{G}_{12}$ species were detected in solution, probably because of their rapid elongation. To take into account the monodisperse length of the quadruplexes, two additional processes were included in the model: annealing, which favors longer particles, and fragmentation, which favors shorter ones. These processes were considered to balance and determine the final quadruplex length [24].

Quadruplex growth-in-length induced by concentration was demonstrated also by NMR and dynamic light scattering experiments performed on $\mathrm{d}(\mathrm{pG})$ in the form of sodium salt [28]. Two distinct types of aggregate species, consisting of stacked monomers and stacked G-quartets, were detected. Their length was found to increase with concentration but was insensitive to added $\mathrm{NaCl}$. Moreover, the size of Gquartet aggregates was observed to be essentially independent on temperature, in contrast with the common temperature/concentration phase-diagram observed in several guanosine derivatives $[13,19]$.

Temperature dependence of the self-assembly of $d(p G)$ was indeed controversial. At $c=5 \mathrm{wt} \%$, DLS measurements 
on $\mathrm{d}(\mathrm{pG})$ in the form of ammonium salt revealed the presence of self-assembled quadruplexes in addition to large globular aggregates. By increasing temperature, the number of quadruplexes was observed to abruptly decrease [29]. Such an abrupt transition between self-assembled and monomeric objects is however unusual for linear self-assembling systems [30]. DLS experiments were also performed on aqueous solutions of $\mathrm{d}(\mathrm{pG})$ and $(\mathrm{pG})$, both in the form of ammonium salts, in the pretransitional region of the isotropic-tocholesteric phase transition [31]. Even if $d(p G)$ assembling occurs at lower concentrations and longer aggregates form, the aggregate melting curves of both derivatives were very similar. In particular, a low-temperature interactioncontrolled region, an intermediate narrow region of intense aggregate dissociation, and a high-temperature region of dissociated species were identified. Besides, a DLS study on $(\mathrm{pG})$ free acid solutions showed that self-assembled aggregates dissociate gradually on heating, and that the aggregation state of dissociated species is strongly related to guanosine concentration [32].

Due to contradictory findings reported in literature, temperature effects on the $d(p G)$ self-assembling require further investigations. In this work, we report on a series of small-angle X-ray scattering experiments, which have been performed as a function of temperature on aqueous solutions of $\mathrm{d}(\mathrm{pG})$ in the form of ammonium salt. Samples were prepared in the absence of excess salt and at a $d(p G)$ concentration just above the critical concentration at which self-assembling occurs. A global fit approach has been used to derive the composition and size distribution of the scattering particles as a function of temperature. The obtained results give thermodynamical justification for the observed phasebehavior.

\section{Material and Methods}

2'-deoxyriboguanosine 5'-monophosphate (Sigma, St. Louis, USA; $99 \%$ purity), in the form of ammonium salt, was obtained by exchanging sodium/ammonium cations using an Amberlite 200 ion exchange resin (Fluka, Deisenhofen, Germany) followed by subsequent lyophilization. To purify $\mathrm{d}(\mathrm{pG})$, the lyophilized powder was redissolved in pure water, precipitated by adding two or three volumes of $95 \%$ ethanol and collected by centrifugation in a microcentrifuge tube at high speed. The pellet was resuspended in distilled water and analyzed after an equilibration time of 24 hours at room temperature. To avoid the introduction of additional ions that can influence the self-assembling process, no buffers were used to adjust the $\mathrm{pH}$ of the solutions. The $\mathrm{d}(\mathrm{pG})$ weight concentration $c$ of the investigated solutions was $50.8 \mathrm{mg} / \mathrm{mL}$, which corresponds to 5 weight percent or to $0.15 \mathrm{M}$.

Small-angle X-ray scattering experiments were performed at the SAXS beamline at the Elettra Synchrotron (Trieste, Italy) [33]. The wavelength of the incident beam was $\lambda=1.54 \AA$ and the explored $Q$-range extended from 0.05 to $0.4 \AA^{-1}$ ( $Q$ is the modulus of the scattering vector, defined as $4 \pi \sin \theta / \lambda$, where $2 \theta$ is the scattering angle).

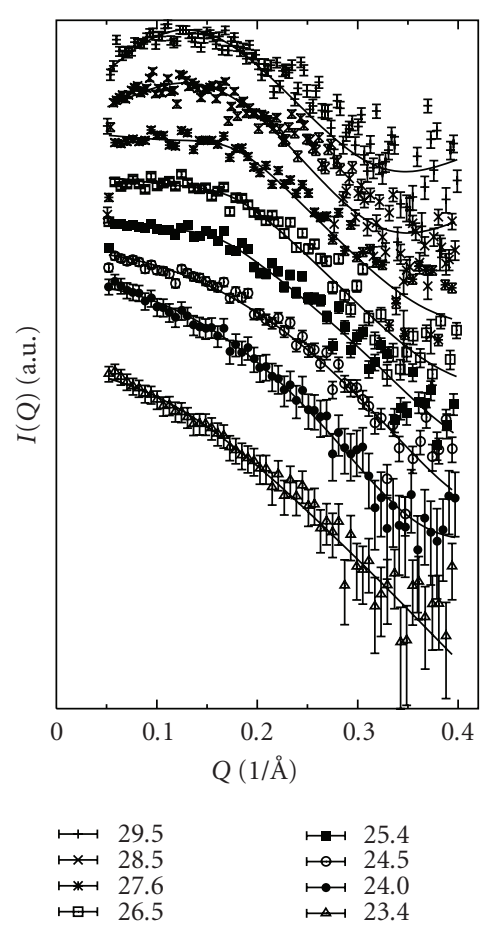

FIGURE 2: In-solution SAXS profiles observed for $\mathrm{d}(\mathrm{pG})$ concentration $c=5 \mathrm{wt} \%$ at different temperatures, as indicated. Best fitting curves obtained using the global fitting approach are shown as solid lines. Note that scattering curves have been scaled by a proper factor.

Guanosine solutions were measured using $1 \mathrm{~mm}$ thick quartz capillaries at different temperatures, from 23 to $30^{\circ} \mathrm{C}$. Particular attention was paid to checking for equilibrium conditions and monitoring radiation damage. In a few tests, measurements were repeated several times (up to 10) at the same temperature to account for a constant scattering signal. Accordingly, to avoid radiation damage the exposure time was $300 \mathrm{~s} /$ frame, while to establish equilibrium condition the dead-time was around 5 minutes. Experimental intensities were corrected for background, solvent contributions, detector inhomogeneities, and sample transmission, as usual [34]. Unfortunately, no absolute scale calibration of the experimental data was available.

\section{Results and Discussion}

SAXS results are reported in Figure 2 and clearly show that the low angle scattering intensity decreases on heating, confirming that the self-assembling process is strongly related to temperature.

3.1. Guinier Analysis. A few structural data, obtained by a simple Guinier analysis of the experimental curves [21], are reported in Table 1. It should be mentioned that in the presence of a chemical equilibrium of different aggregate species (1a)-(1d), the application of the Guiner law can lead to unphysical averaged parameters. Nevertheless, from obtained data a few indications can be extracted. The radius of gyration $R_{g}$ decreases as a function of temperature, 
TABLE 1: Structural data obtained by Guinier analysis of the experimental SAXS curves. $R_{g}$ is the particle gyration radius, while $R_{c}$ is the gyration radius of the particle cross section $[21,35]$.

\begin{tabular}{|c|c|c|c|c|c|c|c|c|}
\hline$T$ & $23.4^{\circ} \mathrm{C}$ & $24.0^{\circ} \mathrm{C}$ & $24.5^{\circ} \mathrm{C}$ & $25.4^{\circ} \mathrm{C}$ & $26.5^{\circ} \mathrm{C}$ & $27.6^{\circ} \mathrm{C}$ & $28.5^{\circ} \mathrm{C}$ & $29.5^{\circ} \mathrm{C}$ \\
\hline$R_{g}(\AA)$ & $11.1 \pm 0.3$ & $8.9 \pm 0.2$ & $7.5 \pm 0.3$ & $6.7 \pm 0.4$ & $5.8 \pm 0.8$ & $5.6 \pm 1.4$ & - & - \\
\hline$R_{c}(\AA)$ & $8.0 \pm 0.1$ & $8.1 \pm 0.8$ & $6.5 \pm 1.5$ & - & - & - & - & - \\
\hline
\end{tabular}

indicating that the size of the aggregates reduces during heating. At temperatures higher than $28^{\circ} \mathrm{C}$, the "melting" process seems to be completed, as no compact particles are any longer detected (in perfect agreement with DLS results obtained at the same concentration [31]). Likewise, the cross section gyration radius $R_{c}$ can be determined only at low temperatures and its value remains rather constant. On one hand, this confirms the presence of cylindrical particles, whose section is fully compatible with the expected crosssection of the guanosine four-stranded helices; on the other hand, it can be inferred that the length of the quadruplexes becomes so short with increasing temperature that the Guiner approximation for tiny rods cannot be applied above $25^{\circ} \mathrm{C}$.

3.2. Global Fitting Analysis. The temperature effects on particle size and composition were then derived by fitting simultaneously the whole set of experimental SAXS curves (8 scattering curves) $[24,36]$. As the $d(p G)$ solution contains randomly oriented scattering particles of different lengths, in equilibrium with each other and dispersed in a homogeneous solvent, the corresponding excess X-ray scattering intensity $I(Q)$ can be written as [35]

$$
I(Q)=\kappa N_{p} P(Q) S_{M}(Q)
$$

where $N_{p}$ is the number density of the scattering particles, $\kappa$ is a calibration factor (which, by comparing the experimental and the nominal sample concentrations, transforms the experimental intensities into scattering cross section in absolute units), and $P(Q)$ and $S_{M}(Q)$ are the effective particle form factor and the measured structure factor, respectively.

For an isotropic system containing particles with different aggregation state, the effective form factor depends on shape, size, and size-distribution of all the particles in solution. In the present case, $P(Q)$ can be written as a weighted sum of the form factors of all the guanosine aggregates eventually present in solution:

$$
P(Q)=\sum_{n=0}^{N} x_{\mathrm{G}_{4 n}} P_{\mathrm{G}_{4 n}}(Q),
$$

where $x_{\mathrm{G}_{4 n}}$ is the number fraction of the $\mathrm{G}_{4 n}$ particle family and $P_{\mathrm{G}_{4 n}}(Q)$ is the corresponding form factor (notice that for $\left.n=0, x_{\mathrm{G}_{4 n}}=x_{\mathrm{G}}\right)$.

The form factor can be calculated from the molecular structure in the case of $\mathrm{d}(\mathrm{pG})$ and $\mathrm{G}$-quartets, while for quadruplexes, a cylindrical model with two-electron density levels and a suitable length has been used (see [24]). On the other hand, $x_{\mathrm{G}_{4 n}}$ is related to both the particle number density $N_{p}$ and the fraction of $\mathrm{d}(\mathrm{pG})$ molecules forming a particular aggregate, $\alpha_{\mathrm{G}_{4 n}}$. In fact,

$$
\begin{gathered}
N_{p}=\frac{10^{-3} N_{A} C}{\left(x_{\mathrm{G}}+4 \sum_{n=1}^{N} n x_{\mathrm{G}_{4 n}}\right)}, \\
\alpha_{\mathrm{G}_{4 n}}=\frac{4 n x_{\mathrm{G}_{4 n}}}{\left(x_{\mathrm{G}}+4 \sum_{n=1}^{N} n x_{\mathrm{G}_{4 n}}\right)},
\end{gathered}
$$

where $N_{A}$ is the Avogadro's number and $C$ is the nominal $\mathrm{d}(\mathrm{pG})$ molar concentration.

$S_{M}(Q)$ is the measured structure factor, related to eventual particle-particle interactions [37]. In dilute solutions, the structure factor is usually negligible $\left(S_{M}(Q) \approx 1\right)$. In the present case, this approximation holds true for SAXS curves obtained below $26^{\circ} \mathrm{C}$. At higher temperatures, the fitting based on $S_{M}(Q)=1$ was very unsatisfactory, even assuming the contemporaneous presence of a variety of different aggregate forms, as stacked G-monomers (observed in [28]) or unstructured particles. Indeed, above $26^{\circ} \mathrm{C}$, particleparticle interactions have to be considered, probably because of the increased number of scattering particles due to the thermal-induced aggregate dissociation. The corresponding $\mathrm{S}_{M}(Q)$ factor was then calculated in the Random Phase Approximation [34, 38], considering the basic approximation of a two-body interaction potential described as the sum of a hard-sphere, a screened Coulombic, and a short-range Yukawian attractive potentials [39]. Details on potentials and form used for the structure factor can be found in [34]; here, it is important to note that the hard-sphere potential depends on the effective particle diameter, $\sigma_{\text {eff }}$, while the main terms determining the screened coulombic and the attractive potentials are the number of charges-per-particle $Z$ and the pair of parameters $J$ and $d$, which correspond to the attractive energy at the particle-particle contact and to its characteristic decay length, respectively [37].

According to (2), data analysis has been then performed by fitting all SAXS curves with an effective form factor, searching for the best structural parameters (namely, radii, electron densities, and length of the cylinders modeling the quadruplexes) and particle composition and concentration, which better described the observed scattering profiles. In agreement with our previous results [24], solutions characterized by the lower number of particle components were preferred. As explained, above $26^{\circ} \mathrm{C}$, we also included a nonzero structure factor.

The GENFIT software was used (see details in [36]). Accordingly, the radius and shell thickness of the cylinder model, the corresponding electron densities, as well as the unique calibration factor $\kappa$ were all considered as global parameters and were therefore obtained from the 


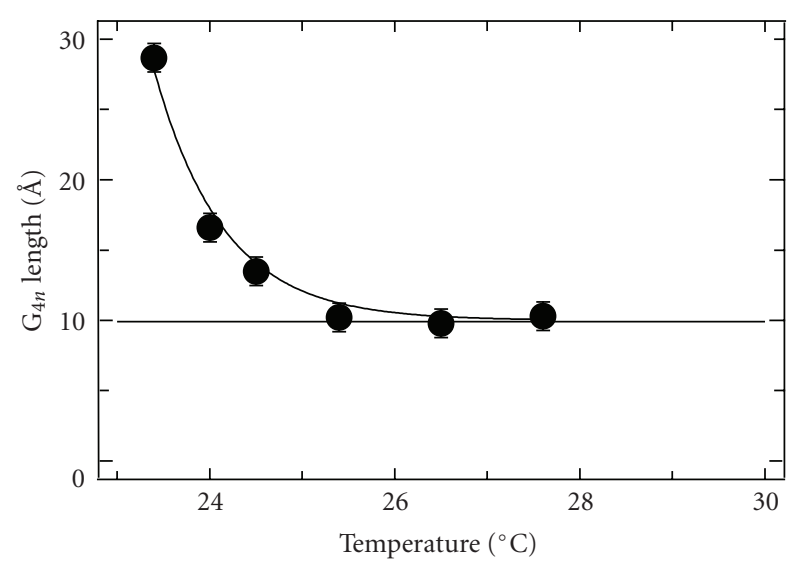

(a)

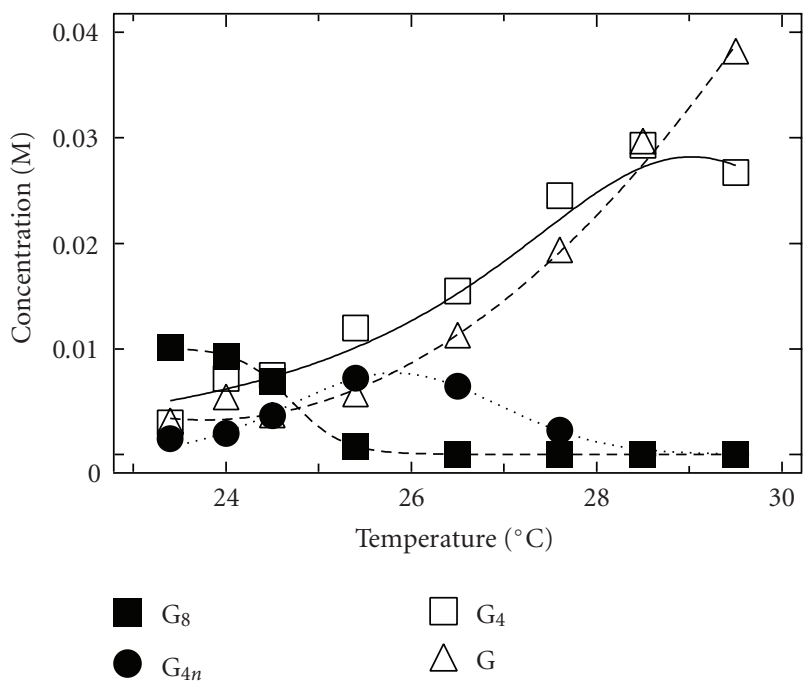

(b)

FIgure 3: Fitting results. (a) Temperature dependence of the length of G-quadruplexes. (b) Temperature dependence of the concentration of the different aggregate species. Symbols are as in the text.

contemporaneous analysis of all the SAXS curves. On the contrary, the pairs $n$ and $x_{\mathrm{G}_{4 n}}$, which are related to the experimental conditions, were obtained from the analysis of each single curve. As dependency of parameters $\sigma_{\text {eff }}, Z, J$, and $d$ on temperature is unknown, they were handled as single curve fitting parameters.

Best-fitting curves are superposed to experimental data in Figure 2, while the fitting parameters are reported in Table 2 and shown in Figure 3 . It can be noticed that all curves are very well reproduced by considering the presence of at least 4 different kinds of scattering particles (namely, free $\mathrm{d}(\mathrm{pG})$ monomers, G-quartets, G-octamers, and monodisperse G-quadruplexes). The use of polydispersion models (as Gaussian, log-normal, or Lorentzian) to describe the quadruplex lengths and the inclusion of particles made by stacked $\mathrm{d}(\mathrm{pG})$ molecules did not improve the fitting results, not even at the higher investigated temperatures.

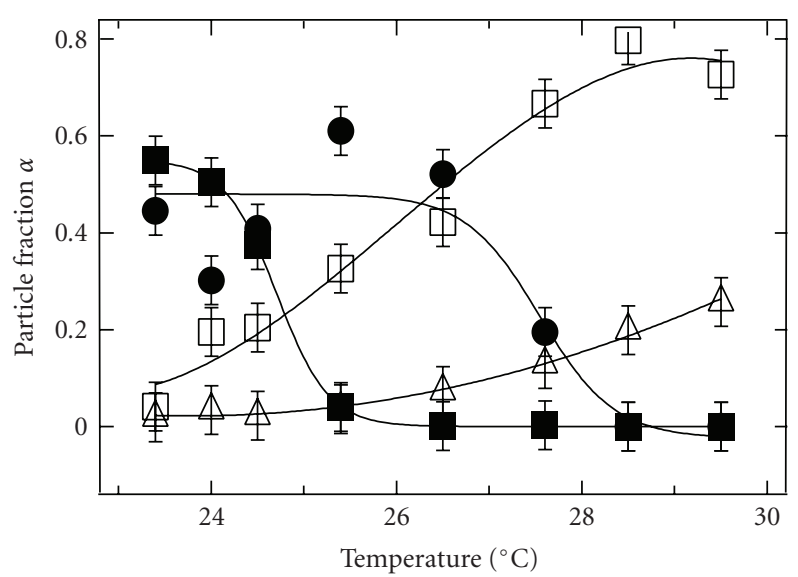

FIGURE 4: Fitting results. Temperature dependence of the fraction of particles assembled in different forms. Symbols are as in Figure 3.

A confirmation of the quality of the global approach is given by the determination of a unique calibration factor: indeed, the whole scattering data have been found to be consistent with the nominal, constant sample composition. Moreover, all the other common parameters are in very good agreement with previous reported structural data $[17-19,21$, $24,25,27]$. As expected, the main structural parameters of Gquadruplexes do not depend on the experimental conditions.

3.3. Temperature Effects on Self-Assembling: Aggregate Structure and Concentration. The temperature dependence of the aggregate composition and of the length of quadruplexes ( $\mathrm{G}_{4 n}$ particles) is reported in Figure 3. Concerning the length of quadruplexes, it can be observed that heating induces particle shortening; however, shortening is rather sharp up to $25^{\circ} \mathrm{C}$, after which a constant length of about $10 \AA$ is reached. This length indicates the presence of dodecamers $\left(G_{12}\right)$. The analysis of the particle composition indicates that octamers are stable only up to $25^{\circ} \mathrm{C}$, while longer particles disappear around $28^{\circ} \mathrm{C}$, with a curious temperature dependence. On the other hand, the amount of both free $\mathrm{d}(\mathrm{pG})$ molecules and G-tetramers continuously increases by heating. Noticeably, the concentration of tetramers starts to decrease at the higher investigated temperatures, even if a "melting" temperature cannot be derived.

The fraction of guanosine molecules assembled in different forms, calculated by (4), is reported in Figure 4 as a function of temperature. Because of compensation between quadruplex length and concentration, the number of guanosine molecules assembled as quadruplexes is rather constant with temperature (or only slighting increasing) until abruptly decreases at some critical point. The corresponding melting temperature is $27.6^{\circ} \mathrm{C}$. In contrast, the fraction of molecules present as $\mathrm{G}_{8}$ shows a temperature dependence which perfectly reproduces the thermal behavior observed for its concentration. An octamer melting temperature of $24.7^{\circ} \mathrm{C}$ is derived by sigmoid data fitting. Therefore, the general features actually indicate the presence of two 
TABLE 2: Single and common parameters obtained by the global-fitting procedure applied to the 8 experimental SAXS curves shown in Figure 2. Symbols are as in the text.

\begin{tabular}{|c|c|c|c|c|c|c|c|c|}
\hline$T\left({ }^{\circ} \mathrm{C}\right)$ & 23.4 & 24.0 & 24.5 & 25.4 & 26.5 & 27.6 & 28.5 & 29.5 \\
\hline$\overline{\sigma_{\text {eff }}(\AA)}$ & - & - & - & - & $8.6 \pm 0.2$ & $9.0 \pm 0.9$ & $9 \pm 2$ & $9 \pm 2$ \\
\hline$Z$ & - & - & - & - & $6.9 \pm 0.3$ & $6.5 \pm 0.5$ & $4 \pm 1$ & $3 \pm 1$ \\
\hline$J(\mathrm{kcal} / \mathrm{mol})$ & 一 & - & - & - & $32 \pm 12$ & $28 \pm 12$ & $30 \pm 12$ & $25 \pm 15$ \\
\hline$d(\AA)$ & - & - & - & - & $2.6 \pm 0.2$ & $2.6 \pm 0.2$ & $1.1 \pm 0.8$ & $1.7 \pm 0.5$ \\
\hline$R(\AA)$ & & & & & $12.2 \pm 0.5$ & & & \\
\hline$r_{\text {core }}(\AA)$ & & & & & $1.9 \pm 0.1$ & & & \\
\hline$\rho_{\text {shell }}\left(\mathrm{e} \AA^{-3}\right)$ & & & & & $0.43 \pm 0.01$ & & & \\
\hline$\rho_{\text {core }}\left(\mathrm{e} \AA^{-3}\right)$ & & & & & $0.325 \pm 0.005$ & & & \\
\hline$\kappa$ & & & & & $(1.6 \pm 0.1) 10^{-3}$ & & & \\
\hline
\end{tabular}

melting processes, that can be attributed to the complex twostep mechanism for guanosine self-assembling. However, the temperature dependence of the fraction of guanosine molecules assembled as tetramers evidences that at high temperature a further melting process occurs: the complete dissociation of G-quartet.

Because the measured structure factor has been calculated considering a number of effective interacting objects in solution [39], the fitted interaction potential parameters should be discussed taking into account the derived particle composition. As shown in Table 2, the average effective particle diameter and the attractive potential parameters can be considered fairly constant within the estimated errors, while the effective particle charge clearly reduces at increasing temperatures. Indeed, in the temperature range where the measured structure factor, and then the interparticle interaction potentials, cannot be neglected (above $26.5^{\circ} \mathrm{C}$ ), the interacting particles are mainly represented by Gquartets, whose dimensions do not depend on temperature. On the other hand, the variation of the effective particle charge is probably related to a temperature-dependent condensation of counter-ions on the particle surface. In particular, the number of total counter-ions presents in solution is essentially determined by the aggregation state, and, for example, octamer disaggregation produces a smaller number of free counter-ions in solution, while tetramer melting increases this number. From (1a)-(1d), it is then clear that temperature will strongly influence the counterion-charge balance. The last comment concerns the values of the two parameters describing the attractive potential. The very small value of $d$ indicates that attraction is short-range. By contrast, $J$ values are surprisingly large, but comparable to interaction energies predicted for G-G stacking $(9.5 \mathrm{kcal} / \mathrm{mol}$ of guanosine [40]) or for the G-quartet stacking in the presence of potassium ( $40 \mathrm{kcal} / \mathrm{mol}$ of $\left.\mathrm{G}_{4}[41]\right)$. Such a result then suggests that the observed short-range attractive term is mainly related to guanosine stacking interactions.

3.4. Temperature Effects on Self-Assembling: Thermodynamics. To derive thermodynamic data on the $\mathrm{d}(\mathrm{pG})$ self-assembling, the behavior described in Figure 3 should be further analyzed. Indeed, two different quadruplex unfolding equilibria, one below and the other above the octamer dissociation

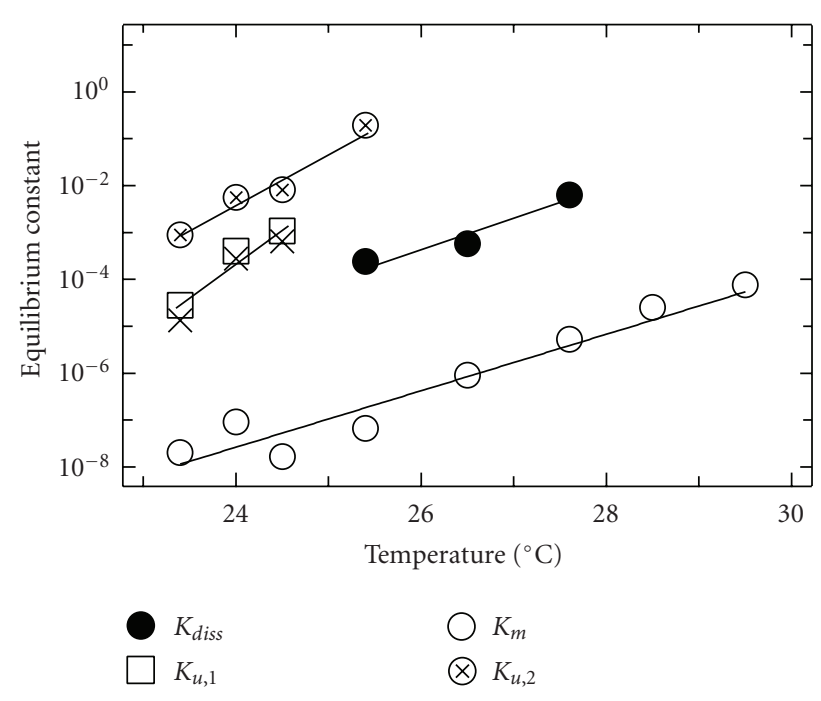

FIGURE 5: Temperature dependence of the equilibrium dissociation constants. Symbols are as in the text. The corresponding reactions are shown in (5).

temperature, exist. In particular, the following dissociation equilibria are suggested to establish at each temperature:

$$
\begin{gathered}
\mathrm{G}_{4 n} \stackrel{K_{u, 1}}{\rightleftharpoons}\left(\frac{n}{2}\right) \mathrm{G}_{8} \stackrel{K_{u, 2}}{\rightleftharpoons} n \mathrm{G}_{4} \stackrel{K_{m}}{\rightleftharpoons} 4 n \mathrm{G} \quad \text { for } T<25^{\circ} \mathrm{C}, \\
\mathrm{G}_{4 n} \stackrel{K_{\text {diss }}}{\rightleftharpoons} n \mathrm{G}_{4} \stackrel{K_{m}}{\rightleftharpoons} 4 n \mathrm{G} \quad \text { for } T>25^{\circ} \mathrm{C} .
\end{gathered}
$$

Equilibrium constants have been derived from aggregate concentrations and their temperature dependence is shown in Figure 5. Note that data concerning quadruplex thermal disaggregation are reported with two different symbols, as they refer to the quadruplex dissociation to octamers (that we identify as the first step in the quadruplex unfolding occurring at low temperature) and directly to tetramers (that we identify as a dissociation process occurring at higher temperature), respectively.

Equilibrium constants increase with temperature, indicating that dissociation of all aggregate species is favored by heating: because equilibrium constant units are different, a direct comparison would be inappropriate, but data 
in Figure 5 suggest that aggregates have different stability (among them, quartets are relatively stable). Noticeable is the strong temperature dependence observed for the equilibrium constant for quadruplex dissociation to octamers, $K_{u, 1}$.

In fact, the increase of $K_{u, 1}$ on heating is higher than those observed for the other equilibrium constants and cannot be explained considering classical temperature effects. A recent analysis on the temperature-dependent self-assembly of $\pi$ conjugated molecules into helical supramolecular fibrillar structures revealed distinct hierarchical stages that govern the formation process [42]. In particular, short oligo $p$ phenylenevinylenes were observed to occur in two different states in dodecane solution, mainly discrete monomeric or hydrogen-bonded dimeric species at high temperature and mainly helical aggregates at low temperature. A nucleationgrowth pathway, based on the Oosawa-Kasai behavior for thermally activated equilibrium polymerization [43], was considered to describe the self-assembling process. In the proposed model, a nonisodesmic helical assembly (characterized by an activation step and subsequent propagation steps) is preceded by an isodesmic assembly of nonhelical preaggregate structures and the two kinds of assemblies are linked through equilibrium between nonhelical and helical assemblies of a critical size. Accordingly, a simple relationship connecting the average aggregation number of the helical assemblies $(N)$ with the equilibrium constants of the elongation process $\left(K_{e}\right)$ and the equilibrium constant of the activation step $\left(K_{a}\right.$, i.e., the equilibrium constant between the active and nonactive state, active meaning the state that can initiate the elongation) was derived [42]:

$$
K_{e}=1-N^{-1}+K_{a} N(N-1) .
$$

A similar aggregate particle thermal profile is observed here (Figure 4); moreover, a nucleation-elongation mechanism was already proposed to analyze SAXS data on guanosine self-assembling as a function of concentration [24]. Therefore, the model presented in [42] has been tentatively applied to describe the thermally induced quadruplex dissociation. In Figure 5, X-shaped symbols indicate the theoretical values for the dissociation constant (i.e., the inverse of the elongation constant $K_{e}$ ) calculated by (6) at any step of quadruplex aggregation (see also Figure 3 ) using $K_{u, 2}$ as reference (note that $K_{u, 2}$ is the inverse of the dimerization constant, and that the formation of octamers is then considered the activation step, i.e., $\left.K_{a}=1 / K_{u, 2}\right)$. The agreement with the experimental data is compelling.

Taking into account the absence of quadruplex elongation above $25^{\circ} \mathrm{C}$, a hierarchical pathway for the self-assembly of guanosine into helical structures upon cooling a solution of dissolved monomers can be proposed. At first, monomers form G-quartets via Hoogsteen hydrogen bonding. On cooling, quartets are brought together via an isodesmic pathway, forming short stacked preaggregates (dodecamers) which, probably, show thermal disorder both in the stacking direction and in the respective quartet orientation. Elongation is not activated. Upon further cooling, the quartes in the preaggregates become more restricted in relative position via a cooperative process. Moreover, at $T \simeq 25^{\circ} \mathrm{C}$, chiral nuclei (octamers) start to form and the elongation-growth pathway sets in. The rapid growth from helical nuclei and helical preaggregates is caused by the many reinforcing noncovalent interactions and is probably enthalpy-driven.

From data interpolated at $25^{\circ} \mathrm{C}$, the free energy changes, $\Delta G_{25^{\circ} \mathrm{C}}$, have been obtained, while plotting data as Van't Hoff isochores $(\ln (K)$ versus $1 / T)$, and under the approximation that standard entropy and enthalpy changes are temperature independent, their values $\Delta H^{0}$ and $\Delta S^{0}$ have been determined. Results are reported in Table 3 . Such a result deserves for a few general comments.

First, free energy changes range around the values observed for the unfolding of quadruplexes made by telomeric or model sequences. $\Delta G$ values extending from 1 to $16 \mathrm{kcal} \mathrm{mol}^{-1}$ per quadruplex, variably depending on loop length or cation, were reported, for example, in $[1,44]$ (data at $20^{\circ} \mathrm{C}$ ) and [45] (data at $27^{\circ} \mathrm{C}$ ), while $\Delta G_{25^{\circ} \mathrm{C}}$ and $\Delta H^{0}$ values per quartet around 2 to $3 \mathrm{kcal} \mathrm{mol}^{-1}$ and 20 to $30 \mathrm{kcal} \mathrm{mol}^{-1}$, respectively, were indicated in [46]. Notice that $\Delta G$ values for quadruplex structures found in literature seem to be constantly higher than values here reported for $\mathrm{d}(\mathrm{pG})$ aggregation. The absence of the covalent axial sugar-phosphate backbone could explain the difference. Second, both $\Delta H^{0}$ and $\Delta S^{0}$ are positive, indicating that the reverse process, namely, the self-assembling, results from the characteristic compensation of favorable enthalpies of formation (around $-30 \mathrm{kcal}$ per mol of guanosine) with unfavorable entropy contributions (around $-130 \mathrm{cal} / \mathrm{K}$ per mol of guanosine). Once more, it is proved that selfassembling is the result of a delicate balance between sometimes opposing forces: the favorable $\Delta H^{0}$ values result from the formation of hydrogen bonds and tetramer stacks, whereas the unfavorable entropies indicate the ordering of the guanines and the uptake of counter-ions and water molecules. Third, thermodynamic parameters provide no evidence (or even provide counter evidence) that tetramer stacking is driven by classical hydrophobic interactions, even if it has often been suggested that this effect is important for quadruplex formation. Fourth, free energy changes associated to quadruplex and octamer unfolding are small and rather similar, and they compare well with the free energy change associated to the dodecamer dissociation. Indeed, $\Delta G_{\text {dis }} \simeq \Delta G_{u, 1}+\Delta G_{u, 2}$, indicating that at $25^{\circ} \mathrm{C}, \mathrm{G}_{4 n}$ and $G_{12}$ forms have the same thermodynamic stability with respect to the G-quartet. However, very different enthalpy and entropy contributions characterize the $\mathrm{G}_{12}$ dissociation: the suggested disorder in the quartet stacking and a binding energy so low that elongation is inhibited are confirmed. Fifth, G-quartets are very stable. Dissociation implies the removal of a coordinated ion. This step is then analogous to the dissociation of an ion from a crown ether, which costs ca. $2.5 \mathrm{kcal} \mathrm{mol}^{-1}$ ion [1], but also includes the favorable free energy of solvation of the counterion and any contingent conformational rearrangement of $\mathrm{G}_{4} \mathrm{M}$ to $\mathrm{G}_{4}$. Accordingly, it is plausible that the energy of counter-ion binding accounts for a substantial fraction of net favorable free energy. Indeed, quartet melting is realized by the breaking of 8 hydrogen bonds. As the strength of a hydrogen bond is between 2 and $10 \mathrm{kcal} \mathrm{mol}^{-1}$ [47], the observed thermodynamic 
TABle 3: Thermodynamic parameters for the different chemical equilibria described by (5). Data are reported on a per mole of guanosine basis.

\begin{tabular}{lccc}
\hline Reaction & $\Delta G_{25{ }^{\circ} \mathrm{C} \mathrm{kcal} \mathrm{mol}}{ }^{-1}$ & $\Delta H^{0} \mathrm{kcal} \mathrm{mol}^{-1}$ & $\Delta S^{0} \mathrm{cal} \mathrm{mol}^{-1} \mathrm{~K}^{-1}$ \\
\hline Quadruplex dissociation to octamers & $0.18 \pm 0.05$ & $37 \pm 8$ & $122 \pm 26$ \\
Octamer unfolding to G-quartets & $0.26 \pm 0.03$ & $45 \pm 6$ & $149 \pm 20$ \\
Quadruplex unfolding to G-quartets & $0.45 \pm 0.05$ & $22 \pm 10$ & $73 \pm 34$ \\
G-quartet melting to free d(pG) & $2.36 \pm 0.04$ & $64 \pm 7$ & $205 \pm 22$ \\
\hline
\end{tabular}

parameters suggest that $\mathrm{H}$-bonds contribute positively to quartet stabilization, but concurrent hydrogen bonds to water and the processes just above discussed should play the main role.

\section{Conclusions}

In order to be able to assess the potential function of G-quadruplexes in a biological system, the self-assembling of deoxyguanosine monophosphate in dilute solution has been studied by SAXS as a function of temperature, from 23 to $30^{\circ} \mathrm{C}$. Samples were prepared in the absence of excess salt, at a concentration of $50.8 \mathrm{mg} / \mathrm{mL}$, just above the critical concentration at which self-assembling occurs [24]. To derive composition and size distribution of the scattering particles, SAXS curves were analyzed by using a global fit approach [36]. As a result, the presence of only a few aggregate forms, namely, free $d(p G)$ molecules, Gquartets, G-octamers, and monodisperse G-quadruplexes, was detected, and their respective concentrations were observed to strongly depend on temperature. In particular, heating induces shortening of G-quadruplexes, that finally disappear around $28^{\circ} \mathrm{C}$, while octamers are stable only up to $25^{\circ} \mathrm{C}$. On the other side, the tetramer melting very probably starts at the higher investigated temperatures, even if a $G_{4}$ "melting" temperature was unfortunately not reached in our experiment.

The thermodynamics of the self-assembling process of $d(p G)$ was further analyzed in the framework of a nucleation-elongation model. The unfavorable nucleation step has been clearly identified with the formation of Goctamers and a hierarchical pathway for the self-assembly of guanosine into helical structures upon cooling proposed. On decreasing temperature, G-quartets form by extended guanosine hydrogen bonding. Through an isodesmic pathway, quartets form stacked dodecamers, which probably show thermal disorder both in stacking direction and in quartet orientation, so that elongation is not activated. Upon further cooling, preaggregates become ordered and chiral nuclei form. The elongation-growth pathway sets in: the rapid growth from the helical nuclei is enthalpy-driven.

To some extent, the picture seems clear, but to derive a complete description of quadruplex formation in the absence of the covalent axial sugar-phosphate backbone, such results should be compared with self-assembling processes occurring in the presence of excess cations, as $\mathrm{Na}^{+}, \mathrm{K}^{+}$, and $\mathrm{NH}_{4}{ }^{+}$. Indeed, concerning the stability of G-quadruplexes formed by oligonucleotides, NMR measurements have shown that the melting kinetics of non-terminal G quartets in a structure containing four G-quartets are very slow (months), whereas they are rapid (minutes) in structures containing only three G-quartets [48]. By comparison, the melting kinetics of Watson-Crick base pairs are in the order of a few milliseconds. Clearly, G-quadruplexes are relatively stable structures, but the number of G-quartets present in a quadruplex dramatically affects its stability. The crucial role that counter-ions could play in driving the formation of stable, long quadruplexes still remains to be investigated.

\section{Acknowledgment}

P. Mariani thanks the Italian MIUR for financial support in the frame of Italia-Slovenia Bilateral project.

\section{References}

[1] A. N. Lane, J. B. Chaires, R. D. Gray, and J. O. Trent, "Stability and kinetics of G-quadruplex structures," Nucleic Acids Research, vol. 36, no. 17, pp. 5482-5515, 2008.

[2] Y. Qin and L. H. H. Hurley, "Structures, folding patterns, and functions of intramolecular DNA G-quadruplexes found in eukaryotic promoter regions," Biochimie, vol. 90, no. 8, pp. 1149-1171, 2008.

[3] S. Chan and E. H. Blackburn, "Telomeres and telomerase," Philosophical Transactions of the Royal Society B, vol. 359, no. 1441, pp. 109-121, 2004.

[4] T. R. Cech, "Beginning to understand the end of the chromosome," Cell, vol. 116, no. 2, pp. 273-279, 2004.

[5] N. I. N. Borovok, D. Zikich, J. Ghabboun, G. I. Livshits, D. Porath, and A. B. Kotlyar, "Assembling of G-strands into novel tetra-molecular parallel G4-DNA nanostructures using avidin-biotin recognition," Nucleic Acids Research, vol. 36, no. 15, pp. 5050-5060, 2008.

[6] H. J. Lipps and D. Rhodes, "G-quadruplex structures: in vivo evidence and function," Trends in Cell Biology, vol. 19, no. 8, pp. 414-422, 2009.

[7] D. J. Patel, A. T. Phan, and V. Kuryavyi, "Human telomere, oncogenic promoter and 5'-UTR G-quadruplexes: diverse higher order DNA and RNA targets for cancer therapeutics," Nucleic Acids Research, vol. 35, no. 22, pp. 7429-7455, 2007.

[8] S. Burge, G. N. Parkinson, P. Hazel, A. K. Todd, and S. Neidle, "Quadruplex DNA: sequence, topology and structure," Nucleic Acids Research, vol. 34, no. 19, pp. 5402-5415, 2006.

[9] M. W. da Silva, "NMR methods for studying quadruplex nucleic acids," Methods, vol. 43, no. 4, pp. 264-277, 2007.

[10] D. Sen and W. Gilbert, "The structure of telomeric DNA: DNA quadriplex formation," Current Opinion in Structural Biology, vol. 1, no. 3, pp. 435-438, 1991. 
[11] A. T. Phan, V. Kuryavyi, and D. J. Patel, "DNA architecture: from G to Z," Current Opinion in Structural Biology, vol. 16, no. 3, pp. 288-298, 2006.

[12] J.-L. Mergny, J.-F. Riou, P. Mailliet, M.-P. Teulade-Fichou, and E. Gilson, "Natural and pharmacological regulation of telomerase," Nucleic Acids Research, vol. 30, no. 4, pp. 839-865, 2002.

[13] G. Gottarelli, G. Spada, and P. Mariani, "The self-assembly of guanosine derivatives and folic acid," in Crystallography of Supramolecular Compounds, Kluwer Academic Publishers, Dordrecht, The Netherlands, 1996.

[14] P. Mariani and L. Saturni, "Measurement of intercolumnar forces between parallel guanosine four-stranded helices," Biophysical Journal, vol. 70, no. 6, pp. 2867-2874, 1996.

[15] P. Mariani, F. Ciuchi, and L. Saturni, "Helix-specific interactions induce condensation of guanosine four- stranded helices in concentrated salt solutions," Biophysical Journal, vol. 74, no. 1, pp. 430-435, 1998.

[16] P. Ausili, M. Pisani, S. Finet, H. Amenitsch, C. Ferrero, and P. Mariani, "Pressure effects on columnar lyotropics: anisotropic compressibilities in guanosine monophosphate four-stranded helices," Journal of Physical Chemistry B, vol. 108, no. 5, pp. 1783-1789, 2004.

[17] F. Federiconi, P. Ausili, G. Fragneto, C. Ferrero, and P. Mariani, "Locating counterions in guanosine quadruplexes: a contrastvariation neutron diffraction experiment in condensed hexagonal phase," Journal of Physical Chemistry B, vol. 109, no. 21, pp. 11037-11045, 2005.

[18] P. Mariani, C. Mazabard, A. Garbesi, and G. P. Spada, "A study of the structure of the lyomesophases formed by the dinucleoside phosphate $\mathrm{d}(\mathrm{GpG})$. An approach by X-ray diffraction and optical microscopy," Journal of the American Chemical Society, vol. 111, no. 16, pp. 6369-6373, 1989.

[19] H. Franz, F. Ciuchi, G. D. Nicola, M. D. Morais, and P. Mariani, "Unusual lyotropic polymorphism of deoxyguanosine-5'monophosphate: X-ray diffraction analysis of the correlation between self-assembling and phase behavior," Physical Review E, vol. 50, no. 1, pp. 395-402, 1994.

[20] C. Detellier and P. Laszlo, "Role of alkali metal and ammonium cations in the self-assembly of the $5^{\prime}$-guanosine monophosphate dianion," Journal of the American Chemical Society, vol. 102, no. 3, pp. 1135-1141, 1980.

[21] F. Carsughi, M. Ceretti, and P. Mariani, "Structural organization of guanosine derivatives in dilute solutions: small angle neutron scattering analysis," European Biophysics Journal, vol. 21, no. 2, pp. 155-161, 1992.

[22] T. J. Pinnavaia, C. L. Marshall, C. Mettler, C. Fisk, H. Miles, and E. Becker, "Alkali metal ion specificity in the solution ordering of a nucleotide, 5' -guanosine monophosphate," Journal of the American Chemical Society, vol. 100, no. 11, pp. 3625-3627, 1978.

[23] A. Wong and G. Wu, "Selective binding of monovalent cations to the stacking G-quartet structure formed by guanosine $5^{\prime}$ monophosphate: a solid-state NMR study," Journal of the American Chemical Society, vol. 125, no. 45, pp. 13895-13905, 2003.

[24] P. Mariani, F. Spinozzi, F. Federiconi, H. Amenitsch, L. Spindler, and I. Drevenšek-Olenik, "Small angle X-ray scattering analysis of deoxyguanosine $5^{\prime}$-monophosphate self-assembing in solution: nucleation and growth of Gquadruplexes," Journal of Physical Chemistry B, vol. 113, no. 22, pp. 7934-7944, 2009.
[25] L. Spindler, I. Drevenšek-Olenik, M. Čopič, et al., "Dynamic light scattering and 31P NMR spectroscopy study of the selfassembly of deoxyguanosine 5'-monophosphate," European Physical Journal E, vol. 7, no. 1, pp. 95-102, 2002.

[26] L. Spindler, I. Drevenšek-Olenik, M. Čopič, and P. Mariani, "Effect of added Ions on the self-assembly of guanosine," Molecular Crystals \& Liquid Crystals, vol. 395, p. 317, 2003.

[27] L. Spindler, I. Drevenšek-Olenik, M. Čopič, J. Cerar, J. Škerjanc, and P. Mariani, "Dynamic light scattering and 31P NMR study of the self-assembly of deoxyguanosine $5^{\prime}$ monophosphate: the effect of added salt," European Physical Journal E, vol. 13, no. 1, pp. 27-33, 2004.

[28] A. Wong, R. Ida, L. Spindler, and G. Wu, "Disodium guanosine $5^{\prime}$-monophosphate self-associates into nanoscale cylinders at $\mathrm{pH}$ 8: a combined diffusion NMR spectroscopy and dynamic light scattering study," Journal of the American Chemical Society, vol. 127, no. 19, pp. 6990-6998, 2005.

[29] L. Spindler, I. Drevenšek-Olenik, M. Čopič, and P. Mariani, "The effect of temperature on the self-assembly of deoxyguanosine $5^{\prime}$-monophosphate in pretransitional region of the I-CH phase transition," Molecular Crystals and Liquid Crystals, vol. 409, pp. 43-50, 2004.

[30] M. P. Taylor and J. Herzfeld, "Liquid-crystal phases of selfassembled molecular aggregates," Journal of Physics: Condensed Matter, vol. 5, no. 17, pp. 2651-2678, 1993.

[31] L. Spindler, F. Federiconi, P. Mariani, et al., "Melting of selfAssembled columnar aggregates formed in aqueous solutions of deoxy- and guanosine 5' -monophosphate," Molecular Crystals \& Liquid Crystals, vol. 435, pp. 661-672, 2005.

[32] H. Jurga-Nowak, E. Banachowicz, A. Dobek, and A. Patkowski, "Supramolecular guanosine 5'-monophosphate structures in solution. Light scattering study," Journal of Physical Chemistry B, vol. 108, no. 8, pp. 2744-2750, 2004.

[33] H. Amenitsch, S. Bernstorff, M. Kriechbaum, et al., "Performance and first results of the ELETTRA high-flux beamline for small-angle X-ray scattering," Journal of Applied Crystallography, vol. 30, pp. 872-672, 1997.

[34] L. R. S. Barbosa, M. G. Ortore, F. Spinozzi, P. Mariani, S. Bernstorff, and R. Itri, "The importance of protein-protein interactions on the $\mathrm{pH}$-induced conformational changes of bovine serum albumin: a small-angle X-ray scattering study," Biophysical Journal, vol. 98, no. 1, pp. 147-157, 2010.

[35] A. Guinier and G. Fournet, Small Angle Scattering of X-Ray, John Wiley \& Sons, New York, NY, USA, 1955.

[36] F. Spinozzi, P. Mariani, L. Saturni, et al., "Met-myoglobin association in dilute solution during pressure-induced denaturation: an analysis at $\mathrm{pH} 4.5$ by high-pressure small-angle $\mathrm{X}$ ray scattering," Journal of Physical Chemistry B, vol. 111, no. 14, pp. 3822-3830, 2007.

[37] F. Spinozzi, D. Gazzillo, A. Giacometti, P. Mariani, and F. Carsughi, "Interaction of proteins in solution from smallangle scattering: a perturbative approach," Biophysical Journal, vol. 82, no. 4, pp. 2165-2175, 2002.

[38] J. Narayanan and X. Y. Liu, "Protein interactions in undersaturated and supersaturated solutions: a study using light and X-ray scattering," Biophysical Journal, vol. 84, no. 1, pp. 523532, 2003.

[39] C. L. P. Oliveira, M. A. Behrens, J. S. Pedersen, K. Erlacher, D. Otzen, and J. S. Pedersen, "A SAXS study of glucagon fibrillation," Journal of Molecular Biology, vol. 387, no. 1, pp. 147-161, 2009.

[40] J. Šponer, H. A. Gabb, J. Leszczynski, and P. Hobza, "Base-base and deoxyribose-base stacking interactions in B-DNA and Z- 
DNA: a quantum-chemical study," Biophysical Journal, vol. 73, no. 1, pp. 76-87, 1997.

[41] A. Calzolari, R. D. Felice, E. Molinari, and A. Garbesi, "Electron channels in biomolecular nanowires," Journal of Physical Chemistry B, vol. 108, no. 8, pp. 2509-2515, 2004.

[42] P. Jonkheijm, P. van der Schoot, A. P. H. J. Schenning, and E. W. Meijer, "Probing the solvent-assisted nucleation pathway in chemical self-assembly," Science, vol. 313, no. 5783, pp. 80-83, 2006.

[43] F. Oosawa and M. Kasai, "A theory of linear and helical aggregations of macromolecules," Journal of Molecular Biology, vol. 4, pp. 10-21, 1962.

[44] C. M. Olsen, H.-T. Lee, and L. A. Marky, "Unfolding thermodynamics of intramolecular G-quadruplexes: base sequence contributions of the loops," Journal of Physical Chemistry B, vol. 113, no. 9, pp. 2587-2595, 2009.

[45] N. Kumar and S. Maiti, "A thermodynamic overview of naturally occurring intramolecular DNA quadruplexes," Nucleic Acids Research, vol. 36, no. 17, pp. 5610-5622, 2008.

[46] V. A. Bloomfield, D. M. Crothers, and I. Tinoco, Nucleic Acids: Structures, Properties and Functions, University Science Book, Sausalito, Calif, USA, 2000.

[47] J. N. Israelachvili, Intermolecular and Surface Forces, Academic Press, London, UK, 1992.

[48] Y. Wang and D. J. Patel, "Guanine residues in d(T2AG3) and $\mathrm{d}(\mathrm{T} 2 \mathrm{G} 4)$ form parallel-stranded potassium cation stabilized G-quadruplexes with anti glycosidic torsion angles in solution," Biochemistry, vol. 31, no. 35, pp. 8112-8119, 1992. 

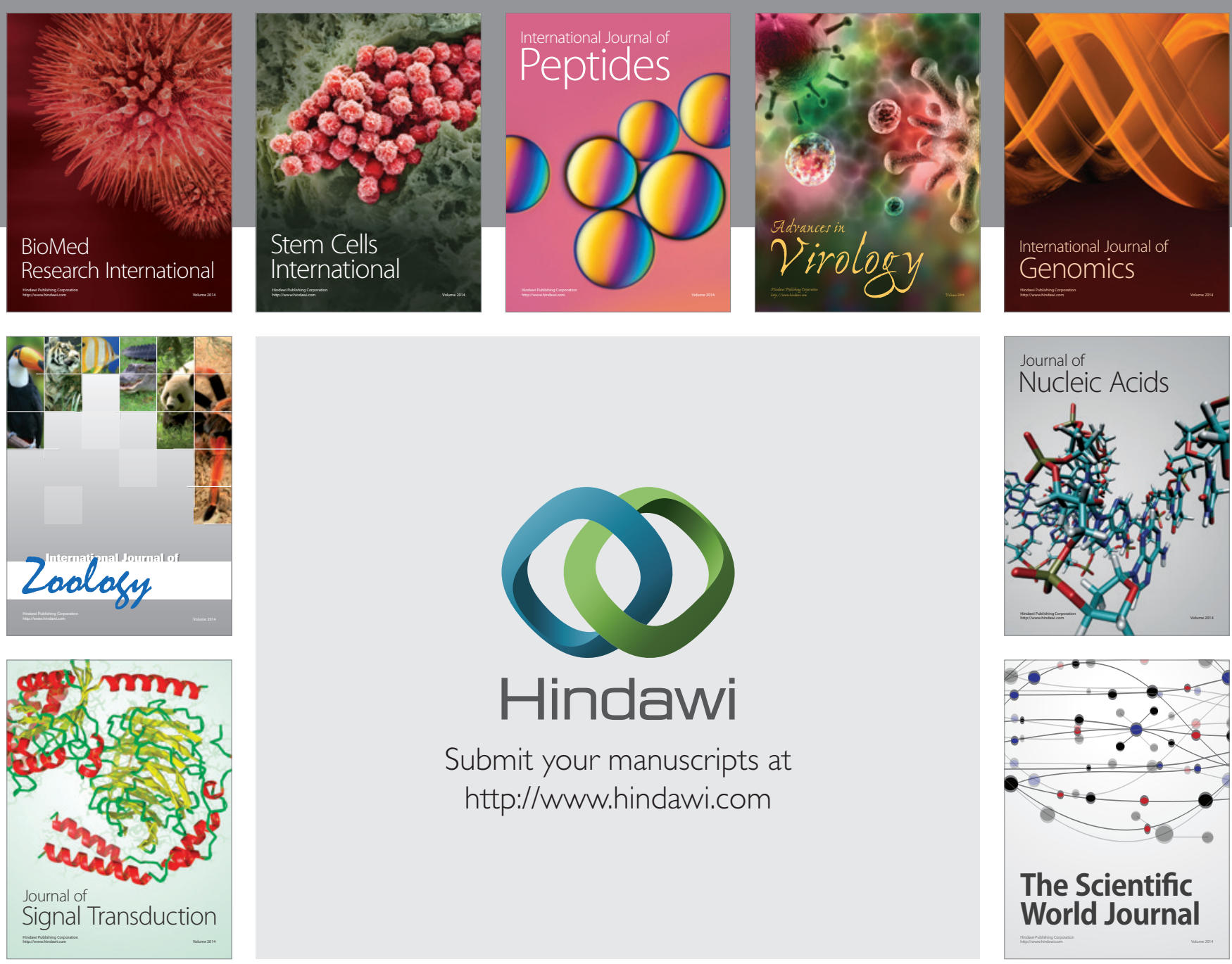

Submit your manuscripts at

http://www.hindawi.com
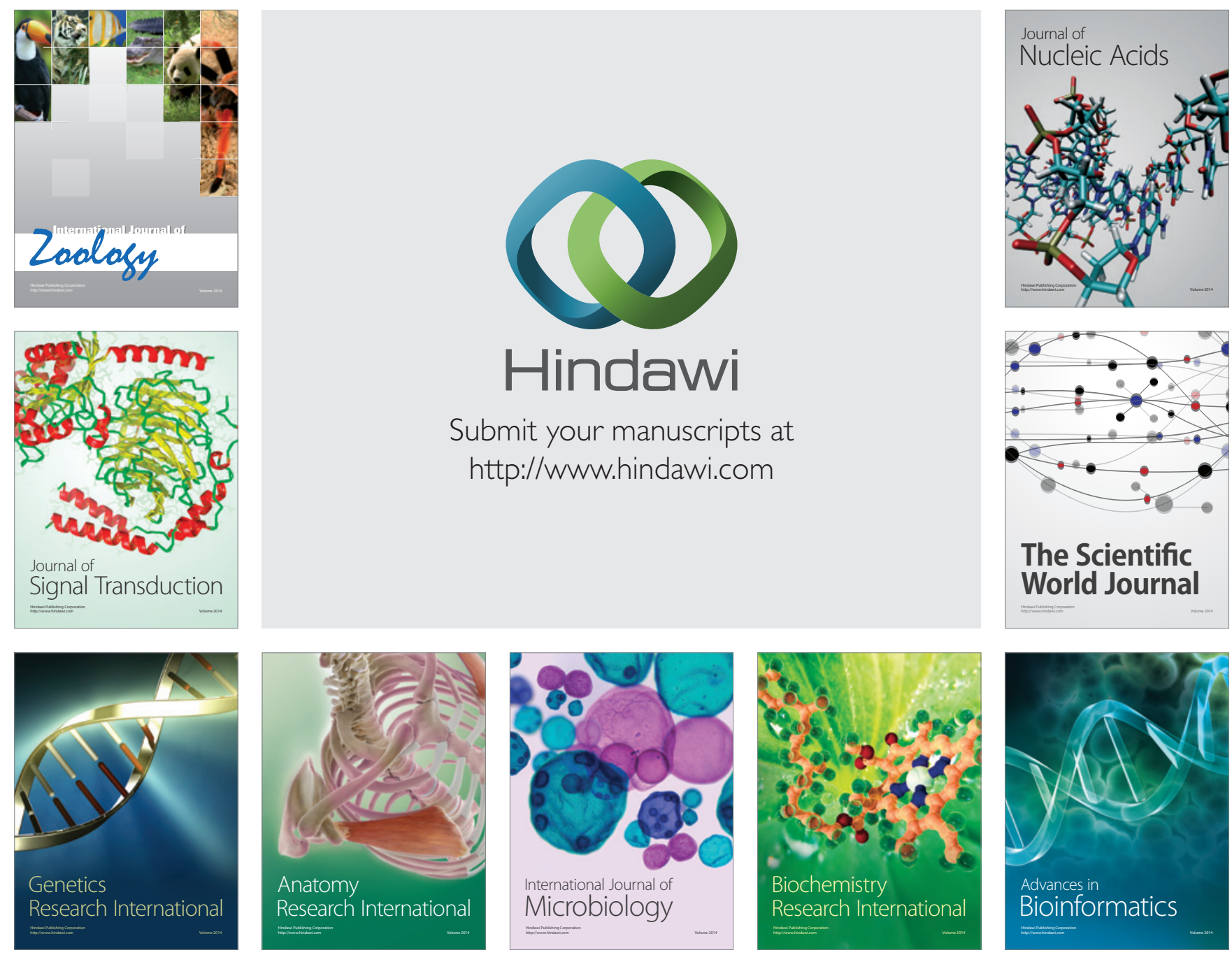

The Scientific World Journal
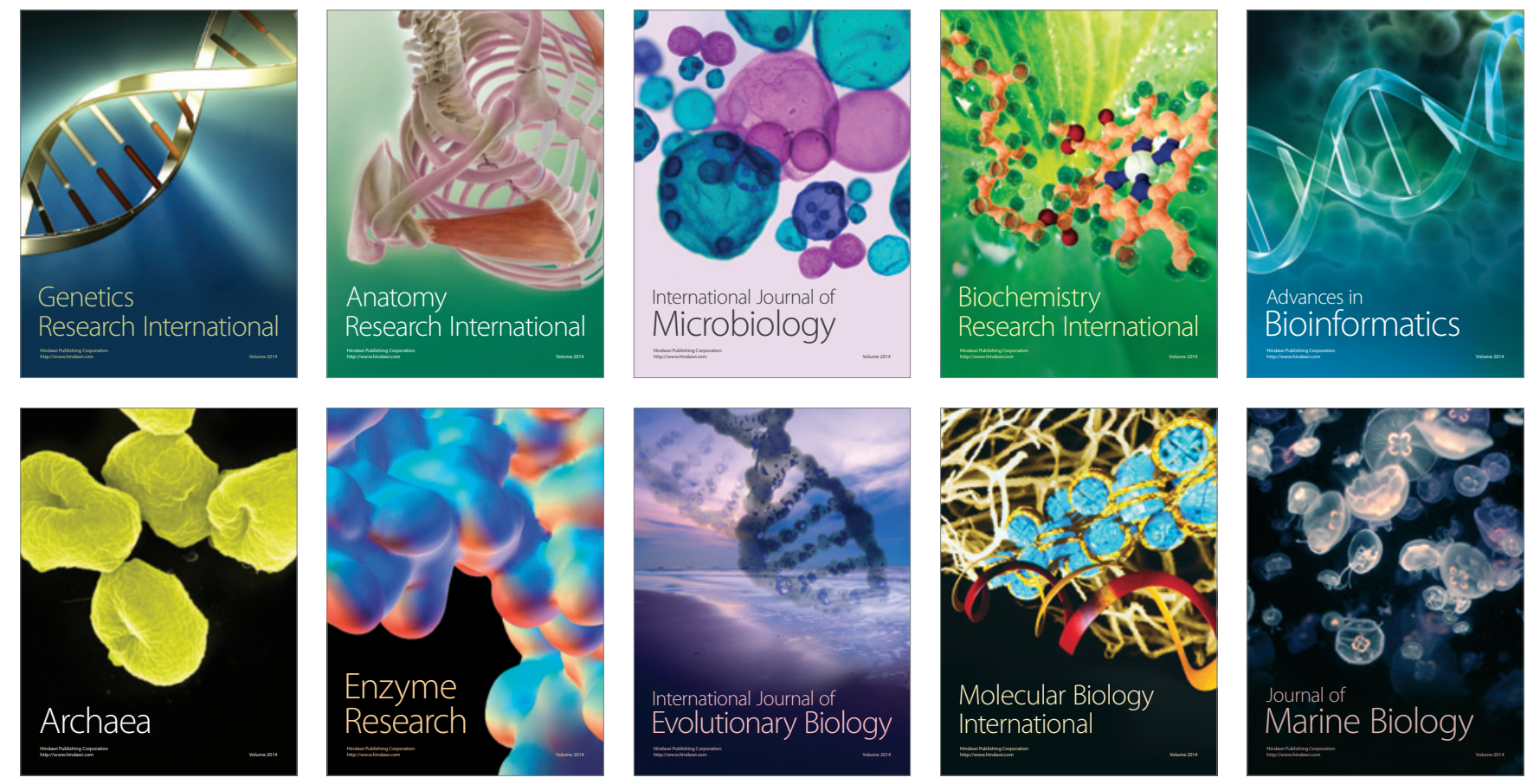